\title{
In vitro Analysis of Antimicrobial Properties of Cefuroxime Impregnated Bone Cement
}

\author{
A Suhail, MS (Ortho), Gunalan*, MS (Ortho), A Sabarul, MS (Ortho), \\ Y Shahril, MS (Ortho), H Salasawati**, O Masbah, MS (Ortho) \\ Department of Orthopaedics \& Traumatology, UKM Medical Centre, Kuala Lumpur, Malaysia \\ *Department of Orthopaedics, Hospital Miri, Sarawak, Malaysia \\ **Department of Microbiology, UKM Medical Centre, Kuala Lumpur, Malaysia
}

\begin{abstract}
Infection after joint replacement surgery is problematic and difficult to treat. The utility of antibiotic laden bone cement for reduction of risk of infection in primary and revision joint replacement surgery has already been established in many studies. In this study, we examined the efficacy of bone cement containing cefuroxime, employing a modified in vitro Kirby-Bauer susceptibility model for investigation of 13 strains of organisms that are found in orthopaedic infections. Organisms investigated were broad spectrum and effective for Gram-positive, Gram-negative, aerobic organisms and anaerobes. Simplex P with added cefuroxime was effective against 8 out of 13 strains. Cefuroxime is stable during exothermic polymerisation of the cement, and is released from the cement at concentrations high enough to inhibit the growth of most organisms encountered after joint arthroplasty.
\end{abstract}

Key Words:

Arthroplasty, Cefuroxime, Antibiotic bone cement

\section{INTRODUCTION}

Since the 1950s, rapid advances have been made in joint arthroplasty, but the progress is somewhat hampered by infections. Increasingly, the use of antibiotic-impregnated bone cement is becoming the standard of practice in Europe and Scandinavia, for both primary and revision knee and hip arthroplasties ${ }^{1}$. The quality of air ventilation and the use of antibiotic impregnated bone cement have led to a stepwise reduction in deep infections ${ }^{2}$. Bone cement mixed with active agents such as antibiotics or anticancer drugs, releases the agents slowly, thus serving as a vector for specific controlled in situ therapy. The primary advantage of antibiotic impregnated bone cement for prophylaxis is the potential for further reduction of an already low rate of deep prosthetic infection ${ }^{3}$.

The current infection rate after primary joint replacement is low, around $1-2 \%$. However the total number is still high in when we consider the total number of joint replacements performed every year ${ }^{4}$. Infection remains a serious problem, as it generally requires multiple surgeries, high cost and/or the patient may end up with a non-functioning joint. At times amputations or mortality become unavoidable in the treatment of these infections.

When compared with systemic therapy, administration of local antibiotics has similar or better effects than systemic administration. Local rinses with antibiotic containing solutions, however, were found to be less effective ${ }^{5}$. Antibiotic-loaded bone cement generally prevents infection of bone cement haematogenously within an hour after implantation, but if the (infectious) challenge is postponed for weeks, this protective effect no longer exists ${ }^{6}$. Cefuroxime is a semisynthetic, broad spectrum cephalosporin antibiotic for parenteral administration that is heat stable so is not denatured or deactivated by heat released during the polymerization process ${ }^{7}$. It can be used prophylactically, is readily available in most regional hospitals and also has no adverse effects in patients with impaired renal function, unlike gentamycin that has the potential of adversely affecting renal function especially in elderly patients or those with already impaired renal function.

The ability to mix cefuroxime to the bone cement on the spot during surgery offers a much cheaper option compared to the pre-mixed antibiotic bone cement. Biomechanical studies have also proven that Cefuroxime impregnated bone cement has similar pullout strength compared to other pre-mixed antibiotic bone cement ${ }^{8}$. We conducted this study to evaluate the diffusion of cefuroxime and its antimicrobial properties when impregnated in bone cement.

\section{MATERIALS AND METHODS}

Under sterile conditions, Simplex (P) bone cement was hand mixed thoroughly with $20 \mathrm{ml}$ of monomer liquid according to the manufacturer's recommendation. Before the setting time the bone cement was inserted into a metal template to produce discs of $14 \mathrm{~mm}$ diameter and $2 \mathrm{~mm}$ thick and was 
then compressed using two solid stainless steel slabs for ten minutes for curing. After curing the discs were tamped free and immediately assayed. The same procedure was repeated with the addition of $1.5 \mathrm{~g}$ of Cefuroxime to another batch of bone cement discs and they were stored separately.

Agar plates were streaked for confluent growth of bacteria included Gram-positive and Gram-negative aerobic organisms 24-48 hours before adding the cement discs as specified below. The ten bacterial strains used were: Staphylococcus aureus, Staphylococcus coagulase negative (CONS), Methicillin Resistant Staphylococcus aureus (MRSA), E. coli, Proteus sp., Enterobacter sp., Pseudomonas aeruginosa, Klebsiella sp., Extended Spectrum Beta Lactamase (ESBL) Klebsiella and Acinetobacter sp. (Table I). Test organisms were propagated and handled in accordance with National Committee for Clinical Laboratory Standards (NCCLS) recommendation for broth media, agar and incubation specifications.

The distribution of the strains of each organism was chosen because of frequency of occurrence in clinical situations. Each organism was cultured onto two agar plates. For each bacterial strain tested, one antibiotic laden bone cement disc was applied to one cultured plate, and one plain cement disc was applied to the second plate of the same strain as a control.

All plates were then incubated at 30 degrees Celsius and examined at 24h, 48h, 72h, 4d, 5d, 7d, 9d, 11d, 13d and 15th day after the application of the cefuroxime laden bone cement or plain bone cement. Zones of inhibition were defined as the distance between the test disc and the edge of bacterial growth. Susceptible strains were defined as those that have a definite zone of inhibition and resistance strains as those without a zone of inhibition, (as seen in all of the control discs) ${ }^{9}$.

\section{RESULTS}

There were zones of inhibition around the antibiotic laden bone cement discs in some agar plates, while in others there was no inhibition at all. The width of the inhibition zone was charted against the specific organisms according to the number of days following the inoculation (Table1). Susceptibility towards antibiotic bone cement is defined as a zone of inhibition of $2 \mathrm{~mm}$ or more .Bacterial strains that showed susceptibility towards Cefuroxime laden bone cement are Staphylococcus aureus, CONS, E coli, Proteus sp., Enterobacter sp. and Klebsiella sp. whereas MRSA, Acinetobacter sp., Pseudomonas sp. ESBL and Klebsiella didn't show any zone of inhibition. All the agar discs showed highest reading for the zone of inhibition in the first few days and a reduction of the zone in the following days for all susceptible organisms. The largest zone of inhibition was seen with Staphylococcus aureus and Staphylococcus coagulase negative at $15 \mathrm{~mm}$. (Figure 1).

Cefuroxime laden bone cement's antimicrobial properties cover certain Gram-positive and Gram-negative organisms quite well but not so for the 'resistant' strains. The activity of cefuroxime bone cement was good even at 15 days after applying it to the growth agar as seen by adequate zones of inhibition still present at the end of day 15 .

\section{DISCUSSION}

The present rate of infection in arthroplasty surgery ranges from $0.5 \%-3 \%{ }^{10}$. Most of these infections are caused by bacteria, thus antibiotics have become an important component of arthroplasty surgery. There are many studies showing that infection rates have been reduced with the use of antibiotic bone cement; In one such study, infection rates were down to $1.6 \%$ from $6 \%{ }^{11}$. Therefore as expected, in many centres antibiotic laden bone cement has become an integral part of arthroplasty surgery.

The advent of new antibiotics and development of resistant bacteria from time to time alter our concepts towards antibiotic impregnated bone cement. Certain criteria, such as the antimicrobial spectrum of activity, should be considered when choosing an antibiotic during arthroplasty. The antibiotic should be safe with minimal side effects, and it should be heat stable so that the heat released during polymerization doesn't cause the antibiotic to lose its effectiveness. The antibiotic should be released from the cement for an extended period of time to provide prolonged antibacterial protection and should not alter or weaken the structure of the bone cement.

Presently, commonly used antibiotics for arthroplasty include: tobramycin, gentamycin, vancomycin, nafcillin, cefalothin, cefazolin, cefotaxime, cefamandole, erythromycin and clindamycin. Each of these antibiotics has its advantages and disadvantages. Tobramycin which is the most common antibiotic used in America has high water solubility, good heat stability, is bactericidal at low concentration, but it is very expensive ${ }^{12}$. Aminoglycosides are known to have a high incidence of ototoxicity and nephrotoxicity. Vancomycin is not effective against Gramnegative bacteria. The penicillin group of drugs are not the drug of choice because they need to be mixed in large proportions and this may weakens the bone cement structure. They are also known to frequently cause hypersensitivity reactions. Most of the cephalosporins have a broad spectrum of antimicrobial action, but are not heat stable and may become ineffective after exothermic polymerization. Erythromycin is bacteriostatic but is known to cause hypersensitivity reactions and needs to be mixed in large amounts and with another antibiotic thus causing the bone cement structure to weaken. 
Table I: Zone of inhibition and day number of culture

\begin{tabular}{|lcccccccccc|}
\hline Organisms & Day1 & Day2 & Day3 & Day4 & Day5 & Day7 & Day9 & Day11 & Day13 & Day15 \\
\hline $\begin{array}{l}\text { Staphylococcus aureus } \\
\text { Staphylococcus coagulase }\end{array}$ & 15 & 15 & 15 & 15 & 14 & 14 & 14 & 14 & 13 & 13 \\
negative (CONS) & 15 & 15 & 15 & 15 & 14 & 14 & 14 & 13 & 13 & 12 \\
Methicillin Resistant & & & & & & & & & & \\
Staphylococcus aureus (MRSA) & - & - & - & - & - & - & - & - & - & - \\
E coli & 12 & 12 & 12 & 12 & 12 & 11 & 11 & 11 & 11 & 10 \\
Proteus sp. & 13 & 13 & 13 & 12 & 12 & 12 & 11 & 11 & 10 & 10 \\
Enterobacter sp. & 9 & 9 & 9 & 8 & 8 & 7 & 7 & 6 & 6 & 5 \\
Pseudomonas aeruginosa & - & - & - & - & - & - & - & - & - & - \\
Klebsiella sp. & 10 & 10 & 10 & 10 & 9 & 9 & 9 & 8 & 8 & 8 \\
ESBL Klebsiella & - & - & - & - & - & - & - & - & - & - \\
Acinetobacter sp. & - & - & - & - & - & - & - & - & - & - \\
\hline
\end{tabular}

1. The numbers above denotes the distance of zone of inhibition in millimetres.

2. The symbol (-) denotes no zone of inhibition at all.

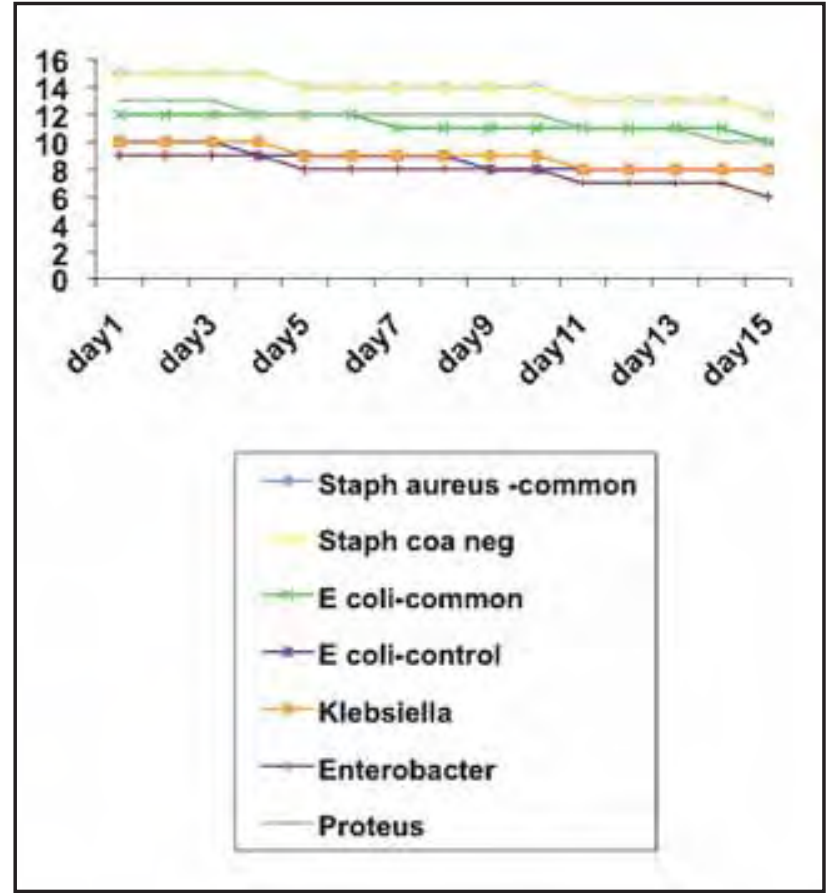

Fig. 1: Zone of inhibition plotted according to days since inoculation of the bone cement

For purposes of this study, we consider an organism to be sensitive to cefuroxime if there was clear zone of inhibition of $2 \mathrm{~mm}$ or more from the edge of the disc. The pattern of sensitivity were consistent with known sensitivities of cefuroxime (Table I), thus confirming that cefuroxime still maintains its antimicrobial properties even after mixing with bone cement and the exothermic reaction during polymerization does not alter its antibiotic properties. Furthermore, it is commonly used as a prophylactic antibiotic in orthopaedic procedures. Generic brands of cefuroxime are already available, which has reduced its cost significantly.

As mentioned earlier the most common antibiotic used as a combination with bone cement is gentamycin. Even though the effect of gentamycin in bone cement is related to classical antibiotic effect, it can have toxic effects due to overdosage ${ }^{13}$. Some authors have also called to stop the usage of aminologlycosides as prophylactic antibiotics. One study reported that aminoglycosides have significant rates of toxicity and resistance, and since there are other antibiotics that demonstrate equal or better efficacies compared to aminoglycosides, its empiric use is not advised ${ }^{14}$.

The outcomes of this study are also supported by two other studies $^{8,15}$. In the first study, 78 osteoarthritis patients in a prospective single blinded randomized study to evaluate the role of cefuroxime impregnated cement in the prevention of deep infection at primary total knee arthroplasty in patients with diabetes mellitus were divided into 2 groups, the first with 41 patients receiving cefuroxime laden bone cement and 37 administered bone cement without antibiotics. With a mean follow-up period of 50 months, results showed that there was no deep infection in group 1 and 5 (13.5\%) deep infections in group 2. $(\mathrm{p}=0.021)$. The author concluded that cefuroxime impregnated bone cement is effective in prevention of deep infection during primary total knee arthroplasty in patients with diabetes mellitus. In the other study, 340 primary total knee arthroplasties were performed with cementless fixation of the femoral component and cement fixation of the patellar and tibial components. The knees were randomly divided into two groups. In Group 1 (178 knees), cefuroxime-impregnated cement was used for fixation, whereas in Group 2 (162 knees), the cement did not contain cefuroxime. The results showed no deep infection developed in the 178 knees in Group 1, whereas a deep infection developed in five (3.1\%) of the 162 knees in Group 2 ( $p=0.0238)$. This author also concluded that cefuroximeimpregnated cement was effective for the prevention of early to intermediate deep infection after primary total knee arthroplasty if used with perioperative systemic antibiotic prophylaxis.

Local release of an antibiotic from cement used to secure an implant may be the most effective means of reaching the 
organisms located at the cement-bone or cement-metal interface as antibiotic laden bone cement provides the highest antibiotic levels in the joint, neighbouring bone tissue, and cement-prosthesis interface during the first 2 weeks after surgery.

\section{CONCLUSION}

This study shows that cefuroxime is heat stable and maintains its antimicrobial properties even after the exothermic reaction associated with polymerization of bone cement. Bone cement impregnated with cefuroxime is effective against Staphylococcus aureus, Coagulase Negative Streptococcus, Proteus sp., Klebsiella sp., E coli, and Enterobacter sp. and beneficial effect last more than two weeks. 


\section{REFERENCES}

1. Buchholz HW, Elson RA, Heinert K. Antibiotic-loaded acrylic cement: current concepts. Clin Orthop 1984; 190: 96-108.

2. McQueen MM, Hughes SP, May P,Verity L,: Cefuroxime in total joint arthroplasty. Intravenous or in bone cement. Journal of Arthroplasty (JOA) 1990; 5(2); 169-72.

3. Harkness JW. Arthroplasty of hip. Campbell's operative orthopaedics. St. Louis: Mosby-Year Book, Inc.; 1998: $296-471$.

4. Berbari EF, Hanssen AD, Duffy MC, Steckelberg JM, Ilstrup DM, Harmsen WS, Osmon DR. Risk factors for prosthetic joint infection: case-control study. Clin Infect Dis 1998; 27: 1247-54.

5. Vehmeyer SB, Slooff AR, Bloem RM, Petit PL. Bacterial contamination in hip and knee arthroplasty. J Bone Joint Surg Br 2000; 82: $561-5$

6. Charnley J. Postoperative infection after total hip replacement with special reference to air contamination in the operating room. Clin Orthop 1972; 87: 167-87.

7. Espehaug B, Engesaeter LB, Vollset SE, Havelin LI, Langeland N. Antibiotic prophylaxis in total hip arthroplasty. Review of 10,905 primary cemented total hip replacements reported to the Norwegian arthroplasty register., 1987-1995. J Bone Joint Surg Br 1997; 79: 590-5.

8. Fuad M D. Biomechanical properties of bone cement with addition of Cefuroxime antibiotic. (Dissertation for Master of Surgery, UKM) 2005.

9. Passuti N and Gouin F. Antibiotic-loaded bone cement in orthopedic surgery. Joint Bone Spine 2003; 70: 169-74.

10. Fitzgerald RH, Hanssen AD. Postoperative deep wound infection. In: Morrey F, editor. Joint Replacement Arthroplasty. Edinburgh: Churchill-Livingstone; 1991: 835-50.

11. Bourne RB.Prophylactic use of antibiotic laden bone cement-An Emerging Standard - In the Affirmative. Journal of Arthroplasty (JOA) 2004 19; (4Supp1): 69-72.

12. William AJ, Arlen DH, Greenwald AS. Antibiotic loaded bone cementin aseptic total joint replacement; American Academy of Orthopedic Surgeons - 17th Annual Meeting 2003.

13. Ruzaimi Bin Mohd Yusoff. In Vitro analysis of antimicrobial properties of Erythromycin and Colistin impregnated bone cement. (Dissertation for Master of Surgery, UKM) 2005.

14. He Y, Trotignon JP, Loty B, Tcharkhtchi A, Verdu J. Effect of antibiotics on the properties of poly(methylmethacrylate)-based bone cement. J Biomed Mater Res 2002; 63:800-6.

15. Gaine WJ, Ramamohan NA, Hussein NA, Hullin MG,McCreath SW. Wound infection in hip and knee arthroplasty. J Bone Joint Surg Br 2000; 82:561-5. 